\title{
Practicas alimentarias y factores asociados al estado nutricional de lactantes ingresados al Programa Alimentario Nutricional Integral en un servicio de salud

\author{
Eating habits and factors associated with nutritional state in \\ infants referred to the Comprehensive Nutritional Food Program \\ at a health center
}

\author{
Pamela Irala', Vanessa González', Susana Sánchez Bernal', José Acosta'
}

\begin{abstract}
RESUMEN
Introducción: Niño/as con desnutrición son usuarios del Programa Alimentario Nutricional Integral (PANI), poco se sabe de sus prácticas alimentarias o condiciones sociodemográficas. Objetivo: Evaluar la asociación de las prácticas alimentarias y variables sociodemográficas con el estado nutricional de lactantes de 6 a 23 meses usuarios al Programa Alimentario Nutricional Integral (PANI). Materiales y Métodos: Estudio de casos y controles, con niños de 6-23 meses del Hospital Materno Infantil de San Lorenzo asignados a 2 grupos: a)Gupo Estudio-GE: 25 niños del PANI (desnutridos(DNT) o riesgo de desnutrir(RD)b)Grupo Control-GC:25 niños eutróficos E), pareados por edad y sexo. Se averiguó sobre alimentación y variables sociodemográficas. Fueron utilizados 2, Mann Whitney, $\mathrm{T}$ Student y regresión logística, $\mathrm{p}<0,05$ fue significativo. Resultados: Previa aprobación de Comité de Ética, fueron evaluados 50 niños, la mediana de edad fue 13,9 meses, el $72 \%$ (18/25 por grupo) eran varones en ambos grupos, 46/50 (92\%) pertenecían a zona urbana. En GE tenían RD 20/25(80\%) niños, DNT 5/20(20\%); en GC todos eutroficos. No hubo diferencias significativas entre grupos, acceso a agua y saneamiento, duración de lactancia materna (LM) exclusiva o inicio de alimentación complementaria. El GE presentó menor promedio de peso de nacimiento que el GC (2724,6 vs 3430,6 gramos; MannWhitney, $\mathrm{p}=0,001)$, menor duración de LM total (7,5 vs 10,7 meses, T Student $\mathrm{p}=0,04)$, menor escolaridad materna $(8,68$ vs 10,4años; T Student $\mathrm{p}=0,03$ ). en el ingreso económico.) Conclusión: Menor peso de nacimiento, escolaridad materna y de tiempo de lactancia materna se asocian a peor estado nutricional del niño, pero ninguna es predictora.
\end{abstract}

Palabras claves: desnutrición, prácticas alimentarias, peso de nacimiento, lactancia materna, escolaridad materna, factores sociodemográficos.

\begin{abstract}
Introduction: Children with malnutrition are users of the Comprehensive Nutritional Food Program (PANI). Little is known about their eating habits or sociodemographic conditions. Objective: To evaluate the association of eating habits and sociodemographic variables with the nutritional status of infants aged 6 to 23 months referred to the Comprehensive Nutritional Food Program (PANI). Materials and Methods: This was a case-control study of children from 6-23 months seen at the San Lorenzo Maternal and Child Hospital, who were assigned to one of two groups: a) Study Group-SG: 25 children of the PANI with malnutrition (MLN) or at risk of malnutrition (ROM) b) Control Group-CG: 25 healthy children $(\mathrm{H})$, matched by age and sex. We reviewed eating and sociodemographic variables. X2, Mann Whitney, T Student and logistic regression were used, $\mathrm{p}<0.05$ was significant. Results: After study approval by the Ethics Committee, 50 children were evaluated. The median age was 13.9 months, $72 \%$ (18/25 per group) were boys in both groups, $46 / 50$ (92\%) came from urban areas. In the SG, 20/25 (80\%) children had MLN, 5/20 $(20 \%)$ were at ROM; in CG all had normal nutritional status. There were no significant differences between groups regarding access to water and sanitation, exclusive breastfeeding duration (EBD) or start of solid feeding. The SG had a lower average birth weight than the CG (2724.6 vs. 3430.6 grams; Mann-Whitney, $\mathrm{p}=0.001$ ), shorter duration of total EBD (7.5 vs. 10.7 months, Student T p = 0.04), lower maternal education (8.68 vs 10.4 years; Student T $\mathrm{p}=0.03$ ) and in lower economic income groups. Conclusion: Lower birth weight, lower maternal education and shorter breastfeeding duration are associated with worsening of a child's nutritional status, but none of these is predictive.
\end{abstract}

Keywords: Malnutrition, eating habits, birth weight, breastfeeding, maternal education, sociodemographic factors.

${ }^{1}$ Universidad Nacional de Asunción. Facultad de Ciencias Químicas. Carrera de Nutrición Humana. San Lorenzo, Paraguay. Correspondencia:Susana Sánchez Bernal Correo: susana.sanchez.ber@gmail.com

Conflicto de interés: Los autores declaran no poseer conflicto de interés

Recibido: 16/07/2019 Aceptado:21/07/2019

DOI: https://doi.org/10.31698/ped.46022019003

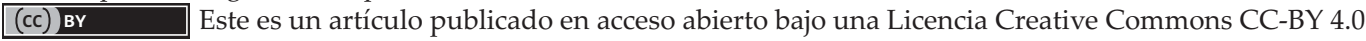




\section{INTRODUCCIÓN}

La alimentación en los primeros 1000 días de vida, correspondientes al periodo desde la gestación hasta los dos años de edad tiene un papel preponderante en el potencial de desarrollo físico, intelectual y emocional del niño ${ }^{(1)}$.

El inicio temprano de la lactancia materna, es decir a la primera hora de vida, protege al recién nacido de las infecciones y reduce la mortalidad neonatal. El riesgo de muerte por diarrea y otras infecciones puede aumentar en los lactantes que solo reciben lactancia parcialmente materna o exclusivamente artificial $^{(2)}$.

Alrededor de los 6 meses de edad, las necesidades de energía y nutrientes del lactante empiezan a ser superiores a lo que puede aportar la leche materna, por lo que se hace necesaria la introducción de una alimentación complementaria. El niño también está suficientemente desarrollado para recibir otros alimentos, los cuales, si no se introducen en ese tiempo o si son administrados de forma inadecuada, el crecimiento del niño puede verse afectado $^{(3)}$.

En la salud del niño interactúan distintos factores, las características en el que las personas nacen, crecen, viven, trabajan y envejecen, incluido el sistema de salud. La OMS los denomina determinantes sociales, que determinan las condiciones en las que un niño será alimentado ${ }^{(4,5)}$.

Una de las consecuencias de una mala alimentación es la desnutrición. Según UNICEF la desnutrición se define como un estado patológico resultante de una dieta deficiente en uno o varios nutrientes esenciales o una mala asimilación de los alimentos ${ }^{(6)}$. Las causas básicas están relacionadas con factores sociodemográficos centrados en la pobreza y la inequidad, (atención sanitaria deficiente, servicios de agua y saneamiento insuficientes y prácticas inadecuadas de cuidado y alimentación $)^{(7)}$.

En el año 2005 se creó en Paraguay el Programa Nacional de Asistencia Alimentaria Nutricional (PROAN) como una estrategia para combatir la desnutrición el cual fue ampliado en el año 2011 pasando a denominarse Programa Alimentario
Nutricional Integral (PANI) orientado a proteger a niños menores de 5 años y embarazadas del flagelo de la desnutrición, siendo actualmente una política pública a través de la promulgación de la Ley 4698/12 “De Garantía Nutricional de la Primera Infancia ${ }^{(8)}$.

Desde el PANI con la entrega del complemento alimentario se contribuye al cumplimiento de los Programas Materno-Infantiles estimulando la asistencia a controles de Salud y favoreciendo así la ejecución de otras medidas de medicina preventiva buscando así un abordaje con una mirada integral del niño y la madre ${ }^{(9)}$.

Estudios demuestran que con el PANI, se logra disminuir significativamente las tasas de desnutrición incluso desde el tercer mes de intervención, también se observó que los usuarios del programa se caracterizan por tener ingreso económico bajo, menor acceso a agua segura y saneamiento básico ${ }^{(10)}$. Sin embargo, también existen niños en las mismas condiciones que no sufren de déficit nutricional evaluado por peso del niño o niña, por lo que el objetivo del presente estudio fue conocer prácticas alimentarias y otros factores de riesgos relacionados con los determinantes sociales de los usuarios del PANI previo al ingreso al programa.

\section{MATERIALES Y MÉTODOS}

Estudio prospectivo de casos y controles. El grupo estudio (GE), estuvo conformado por lactantes de 6 a 23 meses de edad ingresados al PANI y el grupo control (GC), por lactantes sanos sin déficit de peso para edad o talla, pareados por edad y sexo ingresados desde el Hospital Materno entre los meses de mayo y julio del año 2017 previo consentimiento informado y escrito de sus tutores o padres. Fueron excluidos niños portadores o expuestos a VIH, con parálisis cerebral, fibrosis quística, síndrome de Down o con malformaciones congénitas o algún error congénito del metabolismo, así como también a aquellos con antecedentes de alergias o intolerancias alimentarias o con otras patologías de base.

La variable dependiente fue el estado nutricional y 
las independientes estudiadas fueron procedencia, sexo, peso de nacimiento, edad gestacional, tiempo de lactancia materna, edad y escolaridad materna así como también variables sociodemográficos tales como acceso a agua potable, saneamiento básico, ingreso económico, escolaridad materna, así como otros datos sobre la alimentación del niño (edad de inicio de alimentación complementaria o de consumo de otros líquidos diferentes a la leche, entre otros).

La evaluación del estado nutricional se realizo utilizando el puntaje z de Peso para Edad (zPeso/ Edad), puntaje z de Peso para Talla (zPeso/Talla) y el puntaje $z$ Talla para Edad (zTalla/Edad) acorde a los criterios de evaluación de la OMS.

Con una frecuencia de exposición de casos de 0,1 y de 0,5 en los controles, OR a detectar de 2, nivel de seguridad de 0,95 y con potencia de $80 \%$ el tamaño muestral esperado fue de 19 sujetos por grupos (número de controles por caso=1); a fin de salvaguardar posibles pérdidas de sujetos durante el estudio se incorporó un $25 \%$ más alcanzado a 25 niños en cada grupo.

Los datos fueron digitalizados en el programa informático Microsoft ${ }^{\circledR}$ Office Excel 2010 y analizados con el software estadísticos SPSS ${ }^{\oplus}$ versión 20. Las variables continuas con distribución normal se expresaron en medias con desvío estándar y las no homogéneas en medianas con sus valores límites. Las variables cualitativas fueron expresadas en frecuencia o porcentajes. Para la comparación de variables continuas, se empleó la prueba $t$ de Student cuando hubo distribución normal y MannWhitney en caso de ser asimétricas. La asociación entre variables cualitativas se evaluó con prueba de Chi cuadrado. El valor de $p$ designado fue $p<0,05$. Para determinar los factores de riesgo se aplicó el análisis de regresión logística.

Se contó con la aprobación del protocolo de investigación por el Comité de Ética de la Facultad de Ciencias Médicas de la Universidad Nacional de Asunción.

\section{RESULTADOS}

Fueron ingresados 50 niños de ambos sexos con un promedio de edad de 13,9 meses, de los cuales 25 niños pertenecían al grupo estudio y 25 al grupo control. En ambos grupos en su mayoría fueron del sexo masculino $\mathrm{n}=18(72 \%)$ tal como se observa en la Tabla 1, en la que también se presentan los promedios y medianas de puntajes z de los indicadores nutricionales, peso de nacimiento y edad gestacional. Los niños del GE mostraron menor peso al nacer así como menor puntaje zPeso/Edad, zPeso/Talla y de zTalla/Edad.

Tabla1. Distribución por sexo, promedios y medianas de edad, peso al nacer, edad gestacional e indicadores nutricionales.

\begin{tabular}{|c|c|c|c|c|c|}
\hline \multirow[t]{2}{*}{ Variable } & \multirow[t]{2}{*}{ Descripción } & \multicolumn{2}{|c|}{ Grupo estudio Grupo control } & \multirow{2}{*}{$\begin{array}{c}\text { OR } \\
\text { (IC 95\%) }\end{array}$} & \multirow[t]{2}{*}{$\mathbf{p}$} \\
\hline & & $\mathrm{n}=\mathbf{2 5}$ & $n=25$ & & \\
\hline Sexo (n) & Femenino & $7(28 \%)$ & $7(28 \%)$ & & $0,623^{*}$ \\
\hline & Masculino & $18(72 \%)$ & $18(72 \%)$ & $1(0,25-4)$ & \\
\hline $\begin{array}{l}\text { Edad } \\
\text { (meses) }\end{array}$ & $\begin{array}{l}\text { Promedio } \pm \mathrm{DE} \\
\text { (Min-Max) }\end{array}$ & $\begin{array}{c}13,4 \pm 5,4 \\
(6-23)\end{array}$ & $\begin{array}{c}14,1 \pm 5,1 \\
(6-21)\end{array}$ & & $0,98^{* *}$ \\
\hline $\begin{array}{l}\text { Peso Nacimiento } \\
\text { (gramos) }\end{array}$ & $\begin{array}{l}\text { Mediana } \\
\text { (Min-Max) }\end{array}$ & $\begin{array}{c}2900 \\
(740-4250)\end{array}$ & $\begin{array}{c}3416,4 \\
(2590-4100)\end{array}$ & & $0,01 * * *$ \\
\hline $\begin{array}{l}\text { Edad Gestacional } \\
\text { (semanas) }\end{array}$ & $\begin{array}{l}\text { Mediana } \\
\text { (Min-Max) }\end{array}$ & $\begin{array}{c}37,1 \\
(26-42)\end{array}$ & $\begin{array}{c}39 \\
(36-42)\end{array}$ & & 0,22 \\
\hline $\begin{array}{l}\text { zPeso/Edad } \\
\text { (DE) }\end{array}$ & $\begin{array}{l}\text { Promedio } \pm \text { DE } \\
\text { (Min-Max) }\end{array}$ & $\begin{array}{c}-1,92 \pm 0,82 \\
(-4,13--1,14)\end{array}$ & $\begin{array}{c}0,21 \pm 0,70 \\
(-0,96-2,00)\end{array}$ & & $\begin{array}{c}<0,0001 \\
* *\end{array}$ \\
\hline $\begin{array}{l}\text { zPeso/Talla } \\
\text { (DE) }\end{array}$ & $\begin{array}{l}\text { Promedio } \pm \mathrm{DE} \\
\text { (Min-Max) }\end{array}$ & $\begin{array}{l}-1,60 \pm 1,20 \\
(-3,81-1,09)\end{array}$ & $\begin{array}{c}0,05 \pm 0,71 \\
(-1,35-1,25)\end{array}$ & & $\begin{array}{c}<0,0001 \\
* *\end{array}$ \\
\hline $\begin{array}{l}\text { zTalla/Edad } \\
\text { (DE) }\end{array}$ & $\begin{array}{l}\text { Promedio } \pm D E \\
\text { (Min-Max) }\end{array}$ & $\begin{array}{l}-1,48 \pm 1,22 \\
(-3,88-1,08)\end{array}$ & $\begin{array}{l}0,41 \pm 0,96 \\
(-1,32-2,5)\end{array}$ & & $\begin{array}{c}<0,0001 \\
* *\end{array}$ \\
\hline
\end{tabular}

Respecto a la evaluación de la edad gestacional, peso de nacimiento y situación nutricional, en el grupo estudio ningún niño o niña tuvo antecedente de bajo peso de nacimiento, sólo 1 (un) niño fue prematuro y ningún niño presentó desnutrición ya sea por zPeso/Edad, zPeso/Talla o zTalla/Edad tal como se puede observar en la Tabla 2.

En relación con las prácticas alimentarias, en la Tabla 3 se presentan las características investigadas. Se puede destacar que en el grupo estudio, de niños usuarios del PANI la duración total de lactancia materna fue menor, así como también la consistencia de los alimentos de la alimentación complementaria no se adecuaba a las recomendaciones nacionales en cuanto a consistencia al momento del estudio.

Al evaluar factores maternos y sociodemográficos entre ambos grupos, se pudo observar que no hubo diferencias en cuanto a la procedencia, edad materna ni accesos a servicios básicos. Sin embargo, 
en el grupo estudio con niños con déficit nutricional, la escolaridad materna fue menor así como también hubo menor cantidad de familias que acceden al menos a 2 sueldos mínimos tal como se puede observar en la Tabla 4.

Tabla 2. Situación de niños y niñas según peso de nacimiento, edad gestacional y diagnóstico nutricional.

\begin{tabular}{|c|c|c|c|c|c|}
\hline & \multirow[t]{2}{*}{ Descripción G } & \multicolumn{2}{|c|}{ Grupo estudio Grupo control } & \multirow{2}{*}{$\begin{array}{c}\text { OR } \\
\text { (IC 95\%) }\end{array}$} & \multirow{2}{*}{$\begin{array}{l}\text { Valor } \\
\text { de } p^{*}\end{array}$} \\
\hline & & $n=25$ & $\mathbf{n}=\mathbf{2 5}$ & & \\
\hline \multirow{2}{*}{$\begin{array}{c}\text { Peso Nacimiento } \\
N(\%)\end{array}$} & $\geq 2500 \mathrm{~g}$ & $16(64)$ & $25(100)$ & & \\
\hline & $<2500 \mathrm{~g}$ & $9(36)$ & 0 & $7,5(0,77-182)$ & $<0,022$ \\
\hline Prematurez & & $6(24)$ & $1(4)$ & & 0,09 \\
\hline$N(\%)$ & & $19(76)$ & $24(96)$ & & \\
\hline \multirow{2}{*}{$\begin{array}{c}\text { Dx Nutricional } \\
\text { (zPeso/Edad) }\end{array}$} & Desnutridos & $5(20)$ & 0 & & $<0,001$ \\
\hline & Riesgo desnutrir & ir $20(80)$ & 0 & & \\
\hline$N(\%)$ & Eutróficos & 0 & 25 & & \\
\hline \multirow{2}{*}{$\begin{array}{c}\text { Dx Nutricional } \\
\text { (zPeso/Talla) }\end{array}$} & Desnutridos & $7(28)$ & 0 & $24(3-197)$ & $<0,001$ \\
\hline & Riesgo desnutrir & ir $10(40)$ & $2(8)$ & & \\
\hline \multirow[t]{2}{*}{$N(\%)$} & Eutróficos & $7(28)$ & $19(76)$ & & \\
\hline & Sobrepeso & $1(4)$ & $4(16)$ & & \\
\hline \multirow{2}{*}{$\begin{array}{c}\text { Dx Nutricional } \\
\text { (zTalla/Edad) }\end{array}$} & Talla baja & $9(36)$ & 0 & $51(5,4-1203)$ & $<0,001$ \\
\hline & Riesgo talla baja & ja 8 (32) & $1(4)$ & & \\
\hline$N(\%)$ & Talla adecuada & $8(32)$ & $24(96)$ & & \\
\hline Total & & 25 & 25 & & \\
\hline
\end{tabular}

Tabla 3. Características de las prácticas alimentarias de niños de 6 a 23 meses pertenecientes al grupo de estudio y al grupo control.

\begin{tabular}{|c|c|c|c|c|c|}
\hline & & $\begin{array}{l}\text { Grupo } \\
\text { estudio }\end{array}$ & $\begin{array}{l}\text { Grupo } \\
\text { control }\end{array}$ & $\begin{array}{c}\text { OR } \\
\text { (IC 95\%) }\end{array}$ & $\begin{array}{l}\text { Valor } \\
\text { de } \mathrm{p}^{*}\end{array}$ \\
\hline Lactancia materna (alguna vez) & & & \multicolumn{3}{|c|}{$1,5(0,19-15)$} \\
\hline$N(\%)$ & & $22(88)$ & $23(92)$ & & $0,5^{*}$ \\
\hline Lactancia materna exclusiva-LME & & & \multicolumn{3}{|c|}{$0,6(0,14.2)$} \\
\hline (alguna vez) N (\%) & & $20(80)$ & $18(72)$ & & $0,5^{*}$ \\
\hline \multicolumn{6}{|l|}{ Duración de LME (meses) } \\
\hline Media DE & & $3,43 \pm 2,02$ & $3,39 \pm 2,0$ & & $0,652^{* *}$ \\
\hline \multicolumn{6}{|l|}{ Duración de Lactancia materna } \\
\hline total (meses) media DE & & $8,4 \pm 5,2$ & $2,5 \pm 4,9$ & & $0,045^{* *}$ \\
\hline \multicolumn{6}{|l|}{ Edad (meses) en que inició } \\
\hline otros líquidos (agua, té, jugos) & & & & & $0,416^{*}$ \\
\hline Media DE & & $5,23 \pm 2,2$ & $4,33 \pm 2,6$ & & * \\
\hline \multicolumn{6}{|l|}{$\begin{array}{l}\text { Edad (meses) en que inició } \\
\text { alimentación complementaria }\end{array}$} \\
\hline \multirow[t]{3}{*}{ Media DE } & & $5,4 \pm 1,6$ & $5,5 \pm 1,2$ & & $0,63^{* *}$ \\
\hline & Liquido & $1(4)$ & 0 & & \\
\hline & Puré & $22(88)$ & $24(96)$ & & \\
\hline Características de la consistencia & Trocito & $1(4)$ & 0 & & \\
\hline de alimentación complementaria & Combinaciones & es $1(4)$ & $1(4)$ & & \\
\hline \multirow[t]{3}{*}{ de inicio $N(\%)$} & Adecuado & & & & \\
\hline & $\mathrm{Si}$ & $20(80)$ & $25(100)$ & & $0,30 *$ \\
\hline & No & $5(20)$ & 0 & & \\
\hline
\end{tabular}

Tabla 4. Características maternas y de la vivienda, acceso a servicios básicos, e ingreso familiar.

\begin{tabular}{|c|c|c|c|c|c|}
\hline & Descripción & Total & $\begin{array}{l}\text { Grupo } \\
\text { estudio }\end{array}$ & $\begin{array}{l}\text { Grupo } \\
\text { control }\end{array}$ & $\begin{array}{l}\text { Valor } \\
\text { de } \mathrm{p}^{*}\end{array}$ \\
\hline Zona de residencia & Urbana & $48(96)$ & $23(92)$ & $25(100)$ & \\
\hline$N(\%)$ & Rural & $2(4)$ & $2(8)$ & 0 & $0,24^{*}$ \\
\hline Madre con & $\mathrm{Si}$ & $42(84)$ & $22(88)$ & $20(80)$ & \\
\hline pareja & No & $8(16)$ & $3(12)$ & $5(20)$ & $0,35^{*}$ \\
\hline $\begin{array}{l}\text { Edad Materna } \\
\text { (años) media DE }\end{array}$ & & $26,9 \pm 6,7$ & $26,7 \pm 6,6$ & $27,1 \pm 6,9$ & $0,82^{* *}$ \\
\hline $\begin{array}{l}\text { Escolaridad } \\
\text { Materna } \\
\text { (años aprobados) }\end{array}$ & & & & & \\
\hline Media DE & & $9,5 \pm 2,8$ & $8,7 \pm 2,8$ & $10,4 \pm 2,6$ & 0,034 \\
\hline Nivel de Escol. & $\leq 6$ años estudio & $13(26)$ & $10(40)$ & $3(12)$ & \\
\hline Materna N (\%) & $>6$ años estudio & $37(74)$ & $15(60)$ & $22(88)$ & $0,025 *$ \\
\hline $\begin{array}{l}\text { Tipo de agua } \\
\text { para consumo }\end{array}$ & $\begin{array}{l}\text { Agua no tratada } \\
\text { (Pozo) }\end{array}$ & $4(8)$ & $1(4)$ & $3(12)$ & \\
\hline $\begin{array}{l}\text { en hogar } \\
N(\%)\end{array}$ & $\begin{array}{l}\text { Agua tratada } \\
\text { (ESSAP/Aguateria) }\end{array}$ & $46(92)$ & $24(96)$ & $22(88)$ & $0,3^{*}$ \\
\hline $\begin{array}{l}\text { Tipo de baño en } \\
\text { hogar N(\%) }\end{array}$ & $\begin{array}{l}\text { Baño moderno } \\
\text { Letrina con tapa loza }\end{array}$ & $\begin{array}{l}48(96) \\
\quad 2(4)\end{array}$ & $\begin{array}{c}24(96) \\
1(4)\end{array}$ & $\begin{array}{c}24(96) \\
1(4)\end{array}$ & $0,7^{*}$ \\
\hline $\begin{array}{l}\text { Tipo de piso } \\
\text { en hogar }\end{array}$ & $\begin{array}{l}\text { Tierra } \\
\text { Acabado }\end{array}$ & $4(8)$ & $3(12)$ & $1(4)$ & \\
\hline$N(\%)$ & (Cemento/baldosa) & $46(92)$ & $22(88)$ & $24(96)$ & $0,3^{*}$ \\
\hline Paredes del hogar & Ladrillos & $48(96)$ & $24(96)$ & $24(96)$ & \\
\hline$N(\%)$ & Madera & $2(4)$ & $1(4)$ & $1(4)$ & $0,75^{*}$ \\
\hline Ingreso Familiar & $\leq 1$ salario mínimo & $38(76)$ & $22(88)$ & $16(64)$ & \\
\hline
\end{tabular}

\section{DISCUSIÓN}

Este es el primer estudio de tipo caso-control aunque fuera con una muestra pequeña que explora algunos factores que podrían estar asociados con la necesidad de apoyo nutricional debido a bajo peso de niños a tan temprana edad lo que motivó el ingreso al PANI.

Nuestros hallazgos muestran asociación del déficit de peso con el bajo peso al nacer, mayor duración total de la lactancia materna, menor escolaridad materna y con un menor ingreso económico, sin embargo, debido a probable interacción entre variables y al tamaño muestral, no se pudo demostrar la fuerza de predicción de ninguna de las variables estudiadas.

En el grupo de estudio el $24 \%$ de los niños fueron prematuros, mientras que ninguno lo fue en el grupo control, sin embargo, si bien el grupo estudio tuvo niños con menor promedio de edad gestacional que el del grupo control la diferencia no fue significativa. La prematuridad (nacimiento antes de las 37 semanas de gestación), sumada a la desnutrición materna y una ganancia de peso insuficiente 
durante la gestación son las causas más comunes de bajo peso de nacimiento conforme a lo refererido también por Alvarez I. y col (2012) ${ }^{(11)}$.

Respecto al peso de nacimiento de los niños, los niños del grupo estudio (usuarios del PANI) presentaron un promedio significativamente menor al grupo control así como mayor porcentaje de bajo peso de nacimiento, lo cual en nuestro país ya se reportó en el realizado por Sánchez S y col (2014) también con usuarios del PANI en una cohorte, en el que se observó una mayor prevalencia de desnutrición crónica, aguda y global en aquellos niños y niñas con antecedente de bajo peso al nacer $(<2500 \mathrm{~g})$ y peso insuficiente al nacer $(<3000 \mathrm{~g})$ en comparación de los niños con peso igual o superior a $3000 \mathrm{~g}^{(10)}$.

En este contexto, la teoría de Barker que plantea que la nutrición durante el periodo intrauterino y la exposición a infecciones luego del nacimiento determinan la susceptibilidad a enfermar en la edad adulto $^{(12)}$, hace que debamos prestar particular atención a los niños con bajo peso al nacer y a fortalecer la importancia de los primeros 1000 días que incluye el periodo desde la gestación hasta los 2 años de edad ${ }^{(13)}$.

En el studio Women's Health Initiative, así como en varios otros se observó que el peso de nacimiento $<2700$ gramos está asociado de manera independiente con mayor riesgo de enfermedad cardiovascular y que el rol del ambiente y apoyo prenatal y postnatal como contribuyente al desarrollo de enferemedades cronicas no transmisibles ${ }^{(14)}$. De allí la importancia de contar con programas que promocionen adecuadas prácticas de alimentacion y de crianzas, así como también que fortalezcan como el PANI que acoge no sólo a niño y niñas menores de 5 años, sino también a embarazadas en situacion de riesgo nutricional, ya que se ha demostrado una buena aceptabilidad del producto alimentario entregado por el programa ${ }^{(15)}$.

Por otra parte, una de las preguntas del estudio fue si las prácticas alimentarias juegan un rol de preponderancia para determinar el posterior ingreso a un programa de apoyo nutricional como lo es el PANI. No encontramos diferencias en la prevalencia o duración de la lactancia materna exclusiva ni en la edad promedio de inicio de lactancia materna (LM). Del total de niños estudiados el $90 \%$ recibieron alguna vez lactancia materna. En cuanto a la lactancia materna exclusiva se observó que en ambos grupos de estudio no superaban los tres meses, siendo en el grupo estudio de 2,7 meses y en el grupo control de 2,4 meses valores muy similares al trabajo realizado por Sanabria M. y col (2014) en el cual el promedio de lactancia materna exclusiva fue de 2 meses, dicho estudio observó una asociación significativa entre mayor prevalencia de desnutrición crónica y el tiempo de duración de lactancia materna exclusiva menor a 6 meses $^{(16)}$.

Sin embargo, se determinó que los niños del grupo control la lactancia materna total fue más prolongada en su promedio de duración. El 78\% de los niños estudiados recibieron otro tipo de leche a parte de la materna, a causa de esto podría deberse el tiempo reducido tanto de la lactancia materna exclusiva como la del tiempo total de lactancia materna.

La lactancia materna es sin duda el mejor alimento para el lactante menor de 6 meses, ya que posee múltiples beneficios tanto para la madre como para el niño La OMS recomienda el consumo de lactancia materna exclusiva hasta los 6 meses, y el consumo hasta los 2 años acompañado de una dieta variada y equilibrada ${ }^{(17)}$. La LM y la alimentación complementaria no solo desde la perspectiva de crecimiento o desarrollo neurológico, también inciden en el mayor o menor riesgo de obesidad infantil y por ende, de todas las demás afecciones relacionadas con ella ${ }^{(18)}$.

El promedio de inicio de alimentación complementaria en el grupo estudio fue de 5,7 meses y en el grupo control de 5,5 meses, valores muy similares a datos a nivel nacional obtenidos de la Encuesta de ingresos y gastos y de condiciones de vida 2011-2012 donde fue de 5,1 meses ${ }^{(15) .}$

A partir de los 6 meses de edad, las necesidades de energía y nutrientes del lactante empiezan a ser superiores a lo que puede aportar la leche materna, por lo que se hace necesaria la introducción de una alimentación complementaria ${ }^{(3)}$. La introducción de 
los alimentos debe darse de manera paulatina y ser saludables ya que es el tiempo en donde el niño experimenta y descubre nuevos gustos. Estas mismas recomendaciones se encuentran plasmadas en las Guías Alimentarias para niñas y niños menores de 2 años del Paraguay ${ }^{(19)}$.

La edad media de las madres de los niños del grupo estudio fue de 26,6 años, similar a los datos de un estudio de Sánchez S. y col (2017) donde fue 27,5 años $^{(10)}$. La edad materna $<16$ o $>35$ años se asocia en mayor grado a un bajo peso de nacimiento del niño, en consecuencia mayor riesgo a sufrir desnutrición ${ }^{(20)}$. En el presente estudio no fueron incluidas aquellas madres $<18$ años y esto podría ser la razón por la cual no se encontraron diferencias entre ambos grupos de estudio.

Respecto a la escolaridad materna el $40 \%$ de las madres de los niños con desnutrición contaban con 60 menos años de estudio, en un trabajo de Sánchez S. y col (2017) se encontró un valor levemente superior $49,8 \%{ }^{(10)}$. Sin embargo, de igual manera comparando los dos grupos se halló que las madres de los niños con desnutrición tenían un promedio significativamente menor de escolaridad materna frente a los que no presentaban desnutrición (8,6 vs 10,3 años p=0,034). Este hallazgo confirma investigaciones anteriores donde afirman que existe una tendencia al mejor estado nutricional de los menores de edad a medida que el nivel de estudio de las madres aumenta ${ }^{(21)}$.

Las condiciones sociodemográficas son factores que actúan para que las personas presenten diferencias en relación a la posibilidad de ser saludables, mantenerse o tener la opción de recuperar su salud, de ahí la importancia de conocer el ingreso familiar, provisión hídrica, tipo de baño en los cuales no se observaron relación con los casos de desnutrición infantil en el estudio a diferencia de un trabajo similar de Sánchez S. y col (2017) en el cual se encontró un menor porcentaje de desnutrición en hogares que tenían un mejor ingreso económico (1 salario mínimo o más), un sistema sanitario moderno y provisión de agua tratada (ESSAP, Aguateria, red privada) así también no se encontraron diferencias significativas tanto en el tipo de piso, paredes, esto pudo deberse a que el presente estudio se realizó en un solo hospital que se encuentra en la zona céntrica de San Lorenzo y los pacientes que asisten a él pertenecen a la zona de influencia de dicho nosocomio ${ }^{(10)}$.

Los determinantes sociales juegan un rol fundamental como causas estructurales de la desnutrición y sus consecuencias, por lo que pensar que solo el sector salud es responsable de la situación nutricional de la población, es minimizar el contexto en el que se desarrolla la malnutrición. Por ello es fundamental lograr mayor inversión en programas de recuperación nutricional, promoción de la salud y prevención de enfermedades desde un enfoque integral ${ }^{(22)}$.

Al evaluar el estado nutricional de los niños ingresados al PANI en el presente estudio se observó que el $20 \%$ ingresaron con diagnóstico de desnutrición a pesar de ser datos de un solo hospital fueron similares a los datos a nivel país $22,6 \%^{(10)}$ pero es cuatro veces mayor que las cifras nacionales ${ }^{(23)}$ incluso en los publicaciones realizadas respecto a la prevalencia de desnutrición en los servicios públicos de salud ${ }^{(24)}$.

Las limitaciones del presente estudio están dadas principalmente por el tamaño de muestra pequeño que no permitió realizar regresión logística adecuada y que involucra a usuarios que asisten a un hospital público de salud que se halla ubicado en zona urbana, por lo tanto no permite extrapolar la información a una población mayor.

Es por ello que sería necesario aumentar el tamaño muestral así como también involucrar a población del área rural y comunidades indígenas a fin de contar con una mejor aproximación al complejo escenario en el cual se va desarrollando la vulneración de los derechos a un adecuado estado nutricional de la población. Así mismo, es necesario también plantear estudios de cohortes para evaluar en el mediano y largo plazo, los efectos del déficit nutricional de niños y niñas que logran acceder al PANI.

Si bien la implementación del Programa Alimentario Nutricional Integral (PANI) con la entrega de leche mensual a los niños con riesgo a desnutrir o con desnutrición ayuda en gran medida para el tratamiento de la desnutrición ya que es un problema que se observa con mayor frecuencia en 
poblaciones de escasos recursos, pero así también se debe dar apoyo nutricional en la etapa gestacional ya que ese periodo es crítico y determinará la salud de ese niño a corto y mediano plazo. Así también, es crítica la necesidad de un trabajo coordinado con las instancias que inciden en la formulación e implementación de las políticas públicas que afectan positiva o negativamente en los determinantes sociales de la salud y la nutrición.

\section{REFERENCIAS BIBLIOGRÁFICAS}

1. Food and Agriculture Organization of the United Nations. Guias Alimentarias basadas en alimentos [Internet]. FAO; 2016. [Citado 20 de abril de 2016]. Disponible en: http://www.fao.org/nutrition/educacionnutricional/food-dietary-guidelines/background/es/

2. Organización Mundial de la Salud. Alimentación del lactante y del niño pequeño[Internet]. OMS; 2016. [Citado 18 de enero de 2017]. Disponible en: http://www.who.int/ mediacentre/factsheets/fs342/es/

3. Castillo $C$, Balboa P, Torrejón $C$, Bascuñán $K$, Uauyd R. Alimentación normal del niño menor de 2 años. Recomendaciones de la Rama de Nutrición de la Sociedad Chilena de Pediatría 2013. Rev chil pediatr. 2013; 84(5):565-572.

4. Organización Mundial de la salud. Determinantes sociales de la salud [Internet]. OMS; 2017. [Citado 26 de mayo de 2017]. Disponible en: http://www.who.int/soci al_determinants/es/

5. Organización Panamericana de la Salud. Determinantes de Salud [Internet]. OPS; 2017. [Citado 26 de mayo de 2017]. Disponible en: http://www.paho.org/hq/index.php ?option $=$ com_content $\&$ view $=$ article\&id $=5165 \% 3$ A2011determinants-health\&catid $=5075 \% 3$ Ahealthpromotion\&Itemid $=3745 \&$ lang $=\mathrm{es}$

6. UNICEF. Glosario de términos sobre desnutrición. [Internet]. UNICEF; 2017. [Citado 10 de marzo de 2017]. Disponible en: https://www.unicef.org/lac/glosario_maln utricion.pdf

7. Wisbaum W. La desnutrición infantil: causas, consecuencias y estrategias para su prevención y tratamiento. Madrid: UNICEF España; 2011.

8. Instituto Nacional de Alimentación y Nutrición (INAN). Evaluación de programas públicos. Asunción: MSPyBS; 2014.

\section{CONCLUSIÓN}

En relación a las practicas alimentarias se encontró menor duración de la lactancia materna, y menor porcentaje de consistencia adecuada de la alimentación en el grupo estudio en relación al grupo control. Otros factores asociados fueron menor peso de nacimiento, menor escolaridad materna e ingreso familiar en el grupo estudio.

9. Instituto Nacional de Alimentación y Nutrición (INAN). Protocolo de implementación del Programa Alimentario Nutricional Integral-PANI [Base de datos]. Asunción: INAN; 2017.

10. Sánchez S, Aguilar A, Romero L, Cárdenas Y, Romero F, Mendoza L, et al. Evolución Nutricional de niños y niñas menores de 5 años usuarios de un Programa Alimentario Nutricional Integral en Paraguay. Pediatr. (Asunción). 2017;44(1):15-22.

11. Álvarez I, Roca C, Vega M, Rodríguez J, Ruiz Y. Repercusión de la desnutrición materna sobre el nacimiento de niños con bajo peso. MEDISAN 2012; 16(10):1478.

12. Durán P. Nutrición temprana y enfermedades en la edad adulta: acerca de la "hipótesis de Barker". Arch. argent. Pediatr. 2004; 102(1):26-34.

13. Black MM, Walker SP, Fernald LCH, Fernald LCH, Andersen CT, DiGirolamo AM, et al. Early childhood development coming of age: science through the life course. Lancet. 2017; 389(10064):77-90. doi: 10.1016/S01406736(16)31389-7.

14. Smith CJ, Ryckman KK, Barnabei VM, Howard BV, Isasi CR, Sarto GE, et al. The impact of birth weight on cardiovascular disease risk in the Women's Health Initiative. Nutr Metab Cardiovasc Dis. 2016; 26(3):239-45. doi: 10.1016/j.numecd.2015.10.015

15. Cuevas C, Arriola L, Sánchez S. Percepción, prácticas y aceptabilidad de cuidadores de niños/as beneficiarios del Programa Alimentario Nutricional Integral-PANI de 2 hospitales del Departamento Central. Rev. Salud Pública Parag. 2016; 6(2):33-40.

16. Sanabria M, Sánchez S, Osorio A, Bruno S. Análisis de la situación nutricional de los niños menores de cinco años 
en Paraguay a partir de la encuesta de ingresos y gastos y de condiciones de vida 2011-2012. Asunción: UNICEF; 2014.

17. Jimenez R, Aranda E, Aliaga P, Alípaz A, Lopez N, Salazar S, et al. Beneficis nutricionales de la lactancia materna en menores de 6 meses. Rev Med La Paz. 2011; 17(2):5-12.

18. Sandoval L, Jiménez MV, Olivares S, Olvera TDC. Lactancia materna, alimentación complementaria y el riesgo de obesidad Infantil. Aten Primaria. 2016; 48(9):572-578.

19. Ministerio de Salud Pública y Bienestar Social, Instituto Nacional de Alimentación y Nutrición. Guias Alimentarias para la niña y el niño menor de 2 años del Paraguay. Asunción, Paraguay: MSPyBS; 2015.

20. Álvarez I, Roca C, Vega M, Rodríguez J, Ruiz Y. Repercusión de la desnutrición materna sobre el nacimiento de niños con bajo peso. MEDISAN. 2012;16(10):1478.
21. Sobrino M, Gutiérrez C, Cunha A, Dávila M, Alarcón J. Desnutrición infantil en menores de cinco años en Perú: tendencias y factores determinantes. Rev Panam Salud Pública. 2014;35(2):104-12.

22. Richter L, Daelmans B, Lombardi J, Heymann J, Boo FL, Behrman JR, et al. Investing in the foundation of sustainable development: pathways to scale for early childhood development. Lancet. 2016;6736(16):31698-1.

23. UNICEF Paraguay, Dirección General de Encuestas, Estadísticas y Censos, Ministerio de Salud Pública y Bienestar social. Encuesta de Indicadores Multiples por Conglomerado: MICS Paraguay 2016. Asunción, Paraguay: UNICEF, DGEEC, MSPyBS; 2016.

24. Morínigo G, Sánchez S, Sispanov V, Brizuela M, Rolón G, Mendoza L. Perfil Nutricional por antropometría de niños y niñas menores de 5 años del sistema público de salud, 2013. Pediatr. (Asunción) 2015; 42(3):187-191. 